\title{
PRESERVATION OF MICROBIAL SPOILAGE OF FOOD BY BIOSURFACTANT-BASED COATING
}

\author{
VIKRANT SHARMA ${ }^{1}$, MUSKAAN GARG ${ }^{1}$, DEVISMITA TALUKDAR ${ }^{1}$, PALLAVI THAKUR ${ }^{1}$, MARIUS HENKEL ${ }^{2}$, \\ DEEPANSH SHARMA ${ }^{3 *}$, GAURAV KUMAR ${ }^{1 *}$
}

\begin{abstract}
${ }^{1}$ Department of Microbiology, School of Bioengineering and Biosciences, Lovely Professional University, Phagwara, Punjab, India. ${ }^{2}$ Institute of Food Science and Biotechnology, Hohenheim University, Stuttgart, Germany. ${ }^{3}$ Amity Institute of Microbial Technology, Amity University, Jaipur, Rajasthan, India. Email: deepanshsharma@gmail.com/gauravkr@yahoo.com
\end{abstract}

Received: 05 April 2018, Revised and Accepted: 11 July 2018

\section{ABSTRACT}

Objectives: Microbial spoilage of food is one of the leading causes of food scarcity worldwide, which could have devastating effect on the socioeconomic of any country. Along with classical food preservation methods, various innovative approaches can significantly increase the food safety. Biosurfactants are the amphiphilic microbial metabolites, while many of them have potential antimicrobial properties and therefore can be used for food preservation.

Methods: During this study, three biosurfactants obtained from Bacillus subtilis (two of them) and Pseudomonas sp. (rhamnolipid) were screened for their antifungal activity against Aspergillus oryzae (MTCC 1846), Fusarium solani (MTCC 350), and Curvularia sp. by various in vitro and in vivo methods.

Results: During this study, among three surfactants only Pseudomonas sp. biosurfactant (rhamnolipid) exhibited significant antifungal activity against A. oryzae, F. solani, and Curvularia sp. Further, the rhamnolipid coating ( $1 \mathrm{mg} / \mathrm{ml})$ on lemon, potato and tomato protected them from fungal spoilage up to 15 days at room temperature in contrast to untreated samples which started spoiling in 6-7 days.

Discussion: Above findings emphasis on the potential use of biosurfactants for the preservation of food items, however, a detailed study to ensure the safety of biosurfactant is of prerequisite.

Keywords: Biosurfactant, Food spoilage, Rhamnolipid, Amphipathic, Food preservation.

(C) 2018 The Authors. Published by Innovare Academic Sciences Pvt Ltd. This is an open access article under the CC BY license (http://creativecommons. org/licenses/by/4. 0/) DOI: http://dx.doi.org/10.22159/ajpcr.2018.v11s2.28592

\section{INTRODUCTION}

Microbial spoilage of crops and stored food can be disastrous to the socioeconomic of a nation and fungus is one of the most prominent reasons of food scarcity globally. Controlling the fungal rot is a difficult task because once it is initiated, then it is difficult to contain. Around $20 \%-30 \%$ of the total fruit and vegetables produced each year gets lost because of food spoilage $[1,2]$. Extended use of chemical to control the diseases had led to the devastating effect on the environmental balance of microbes in the soil, resulting in the development of resistant strains, groundwater contamination, and risk to human health. The biggest ecological challenge for microbiologists is the development of environmental-friendly substitute for the currently used chemical pesticides for the food preservation. According to many researchers, biological control of the plant pathogens is becoming a trend due to increased pollution concerns because of extensive use of the pesticides, food preservation, and development of the resistant pathogenic strains [3]. Environment-friendly microorganisms are used and have proved to promote plant growth and disease control in modern agriculture. Microorganisms are known to suppress the soilborne plant pathogens through the secondary metabolites including antibiotics. Several studies reported that a variety of microorganisms produces a wide range of surface-active compounds (SAC), known as biosurfactant with fungicidal properties for various fungal species [4].

Microbial surface-active compounds are diverse molecules produced by different microorganism and are classified on the bases of their chemical structure and microbial origin [5]. The major classes of SAC are surfactin, fengycin, iturin, lichenysin, and rhamnolipid compounds with potent antimicrobial, antiadherent, anti-inflammatory, anticancer, antifebrin clot formation, antiviral, antimycoplasma, and hypocholesterolemic activities for a large spectrum of application in medical and pharmaceutical field [6]. They also have application in the field of agriculture as biocontrol, bioremediation, and metal sequestering, also in food processing industries of their emulsifying, foaming, and dispersing properties $[7,8]$. One of the widely studied biosurfactants over the years has been the rhamnolipids [9] and has also reported to show antimicrobial and antifungal properties [10]. One other study reported the application of rhamnolipid biosurfactant against Fusarium sacchari [11]. However, the use of biosurfactant as antifungal agent against several other fungal species has not reported. Therefore, the present study was carried out with the objective to assess the efficacy of biosurfactant as potential fungicidal agent against Aspergillus oryzae, Curvularia sp., and Fusarium solani.

\section{METHODS}

Chemicals

For the in vitro study of the antifungal efficiency (sodium dodecyl sulfate) was used as positive control and procured from Loba Chemie, India. Fungal culture mediums including potato dextrose broth (PDB) and potato dextrose agar (PDA) were procured from Hi-Media Laboratories Pvt., Ltd., India. The biosurfactant isolated in earlier study (data unpublished) from Bacillus subtilis (two of them) and Pseudomonas sp. (rhamnolipids) was used for carrying out the studies.

\section{Phytopathogenic fungal strains}

The phytopathogens A. oryzae (MTCC 1846) and F. solani (MTCC 350) were procured from microbial type culture collection and gene bank (MTCC), Chandigarh. The obtained cultures were revived in the PDB, and then after growth came in PDB, the cultures were transferred on the PDA. Curvularia sp. were isolated from the spoiled chili on the PDA 
plate and purified by repeated subculturing. The identification of the isolated fungi was performed based on its culture characteristics and microscopic structure [12]. The fungus was maintained in PDA and glycerol stocks for further use.

\section{In vitro antifungal activity of the biosurfactant}

The targeted fungal samples including A. oryzae (MTCC 1846), Curvularia sp., and F. solani (MTCC 350) were treated with three biosurfactants including B. subtilis $10 \mathrm{~T}, B$. subtilis 3285 , and Pseudomonas sp. rhamnolipid. The experiment was carried out in the $2 \mathrm{ml}$ sterilized centrifuge tube, where $500 \mu \mathrm{l}$ of biosurfactant solution $(1 \mathrm{mg} / \mathrm{ml}$ in distilled water) was directly inoculated with the mycelium and spores of targeted fungi. Further, the setup was incubated at $28^{\circ} \mathrm{C}$ for $24 \mathrm{~h}$ and the $100 \mu \mathrm{l}$ of the mixture was transferred to the PDA plate from each treatment. The plates were incubated at $28^{\circ} \mathrm{C}$ for $72 \mathrm{~h}$ and were observed for the presence of fungal growth.

\section{In vivo antifungal activity of the biosurfactant}

\section{Vegetable host selection}

Fungicidal activity of the biosurfactant was checked against specific fungi on their suitable host vegetable. During the study, lemon was used for A. oryzae (MTCC 1846), tomato was used for Curvularia sp., while potato was used for $F$. solani (MTCC 350). The selected vegetables were free from wounds and rots, also the selection was based on the homogenous maturity and size.

\section{Antifungal activity of the biosurfactant}

Mature tomato and potato tubers were selected carefully on the absence of any disease or wound and also on the basis their size. The fruits and vegetables were surface disinfected by dipping in sodium hypochlorite $2 \%$ for 10 min followed by rinsing 3 times by sterilized distilled water to eliminate the saprophyte pathogen present on the surface and residual sodium hypochlorite. Then, they were surface dried under the filtersterilized air flow. Further, one lemon was coated with the biosurfactant solution $(1 \mathrm{mg} / \mathrm{ml}$ in water) while the other one was kept uncoated. Both lemons were then stabbed with sterilized wooden stick (Diameter $1.5 \mathrm{~mm}$ ) and the area was sprinkled with spores of A. oryzae (MTCC 1846 ) and incubated at $28^{\circ} \mathrm{C}$ for 15 days. After the incubation, the surface of the lemons was observed for any abnormalities and fungal growth. Later, these lemons were sliced in two equal half and observed for the spread of fungal infection. Similar setup was made to study the antifungal activity of biosurfactant toward F. solani (MTCC 350) and Curvularia sp., while potato and tomato were used, respectively, as host material.
In another setup, biosurfactants coated vegetables $(1 \mathrm{mg} / \mathrm{ml}$ in water $)$ were sprinkled with fungal spores without stabbing the surface of vegetables. Above setup was also incubated for 15 days and observed for any abnormalities and fungal growth on the surface. Later, these vegetables were sliced in two equal half and observed for the spread of fungal infection.

\section{RESULTS}

\section{In vitro antifungal activity of biosurfactant}

The antifungal assay of the biosurfactant was done through challenge method and was checked against $F$. solani (MTCC 350), Curvularia sp., and A. oryzae (MTCC 1846). The targeted fungal mycelium and the spores were treated directly with the biosurfactant solution $(1 \mathrm{mg} /$ $\mathrm{ml}$ ) for $24 \mathrm{~h}$ and later inoculated on PDA plates. The assay was carried out using three biosurfactants including $B$. subtilis (two verities) and Pseudomonas sp. rhamnolipid. Among three biosurfactants, only rhamnolipid exhibited antifungal activity and completely inhibited the growth of all three test fungi on PDA plate. Results of the same are summarized in Fig. 1. Since rhamnolipid showed the desirable activity, it was selected for the further studies.

\section{In vivo antifungal activity on stabbed vegetables}

The in vivo analysis of antifungal potential of the biosurfactant was checked by infecting the fresh vegetables with their respective phytopathogen. The study was carried out on lemon, tomato, and potato where lemon was infected by A. oryzae (MTCC 1846), tomato was infected by Curvularia sp., and potato was infected by F. solani (MTCC 350). The vegetables were stabbed before dusting the fungal spores on them. The experiment was carried out in normal room condition where one fruit sample was surface coated uniformly with the rhamnolipid solution of concentration $1 \mathrm{mg} / \mathrm{ml}$, while the other was kept uncoated. The observations were made after 15 days, and it was observed that the sample which was coated with the biosurfactant solution shown no sign of visible spoilage on surface. The transverse section of the samples was also observed; internally, the coated fruit sample was healthy while the uncoated one was spoiled internally (Figs. 2-4).

\section{In vivo antifungal activity on unstabbed vegetables}

The study was carried out on lemon, tomato, and potato where tomato was infected by Curvularia sp., and potato was infected by F. solani (MTCC 350). The vegetables were not stabbed before dusting the fungal spores on them. The experiment was carried out in normal room condition where one fruit sample was surface-coated uniformly with the rhamnolipid solution of concentration $1 \mathrm{mg} / \mathrm{ml}$, while the other was kept uncoated. The observations were made after 15 days, and it

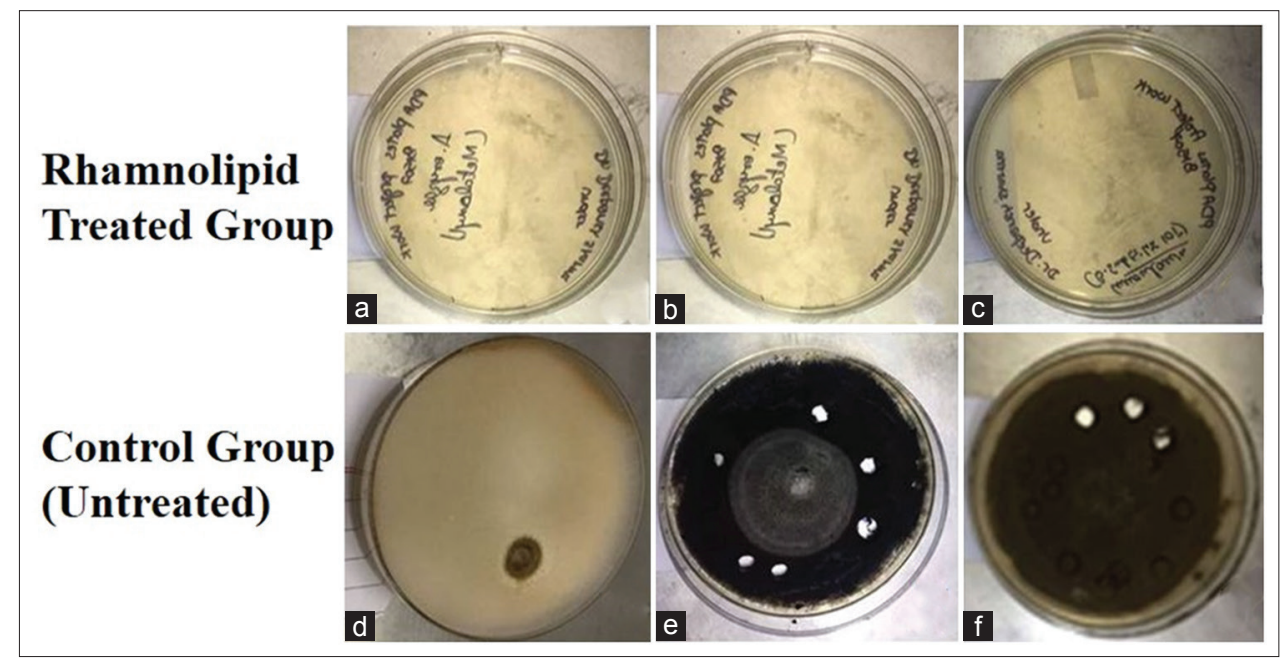

Fig. 1: In vitro antifungal activity of Pseudomonas sp. rhamnolipid. Here, (a-c) are rhamnolipid-treated plates of Fusarium solani (MTCC 350), Curvularia sp., and Aspergillus oryzae (MTCC 1846), respectively; (d-f) are untreated plates of $F$. solani (MTCC 350), Curvularia sp., and A. oryzae (MTCC 1846), respectively 


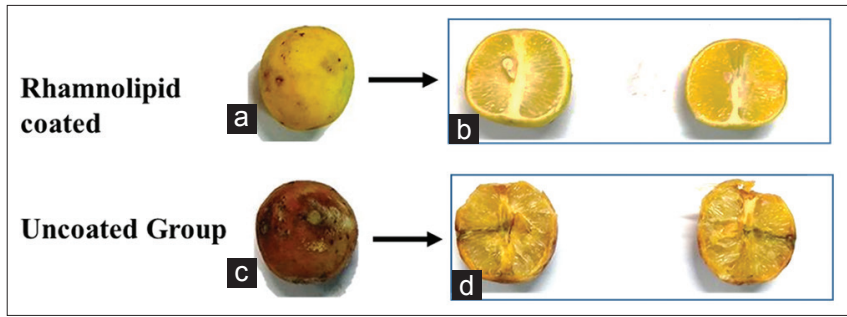

Fig. 2: In vivo antifungal activity of rhamnolipid by surface coating of lemon against Aspergillus oryzae (MTCC 1846) after 15 days. Here, (a) is the rhamnolipid-coated lemon after 15 days; (b) is showing the interior of the rhamnolipid-coated lemon after 15 days; (c) is the uncoated lemon after 15 days; (d) is showing the interior of the uncoated lemon after 15 days

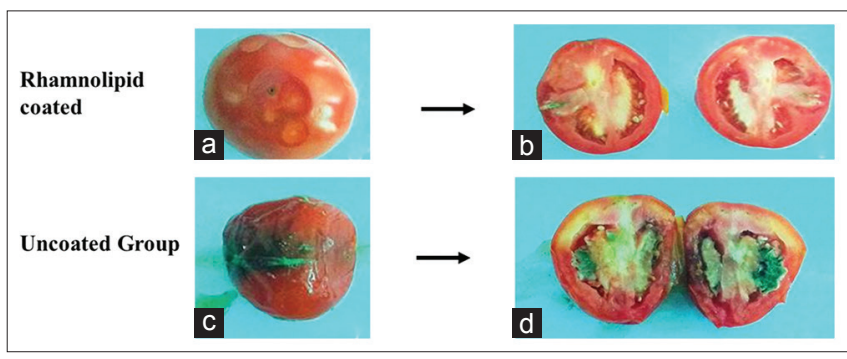

Fig. 3: In vivo antifungal activity of rhamnolipid by surface coating of tomato against Curvularia sp. after 15 days. Here, (a) is the rhamnolipid-coated tomato after 15 days; (b) is showing the interior of the rhamnolipid-coated tomato after 15 days; (c) is the uncoated tomato after $\mathbf{1 5}$ days; (d) is showing the interior of the uncoated tomato after 15 days

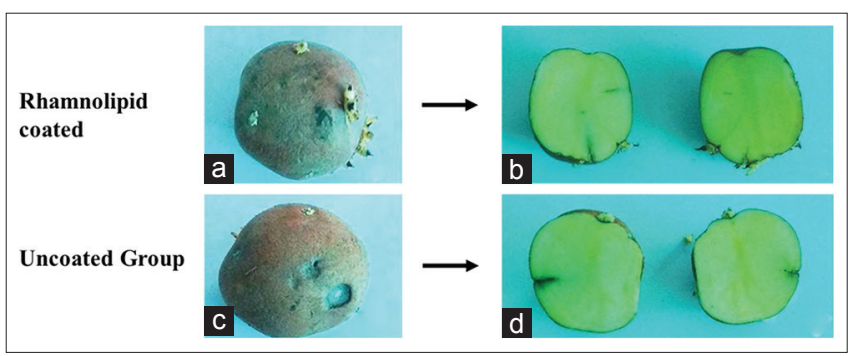

Fig. 4: In vivo antifungal activity of rhamnolipid by surface coating of potato against Fusarium solani (MTCC 350) after 15 days. Here, (a) is the rhamnolipid-coated potato after 15 days; (b) is showing the interior of the rhamnolipid-coated potato after 15 days; (c) is the uncoated potato after 15 days; (d) is showing the interior of the uncoated potato after 15 days

was observed that the sample which was coated with the biosurfactant solution shown no sign of visible spoilage on surface. The transverse section of the samples was also observed; internally, the coated fruit sample was healthy while the uncoated one was spoiled internally (Figs. 5 and 6).

It was also observed that the life span of the fruit sample is also increased, lemon and tomato get easily spoiled within 4-5 days when kept in normal conditions but after the application of the biosurfactant solution, the life span increased to 15 days.

\section{DISCUSSION}

Food spoilage by microorganisms is one of the most prominent regions being the shortage of food worldwide. Studies say that by 2050, we will be requiring an additional of $70 \%$ food and it will continue to upsurge due to increase in population. Currently, a large number of

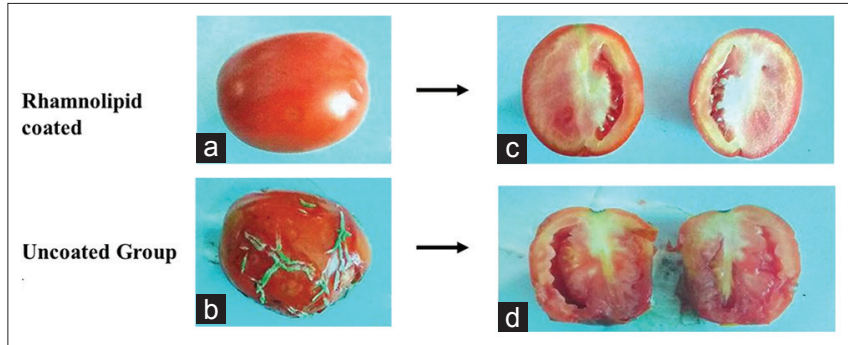

Fig. 5: In vivo antifungal activity of rhamnolipid by surface coating of tomato against Curvularia sp. after 15 days. Here, (a) is the rhamnolipid-coated tomato after 15 days; (b) is showing the interior of the rhamnolipid-coated tomato after 15 days; (c) is the uncoated tomato after $\mathbf{1 5}$ days; (d) is showing the interior of the uncoated tomato after 15 days

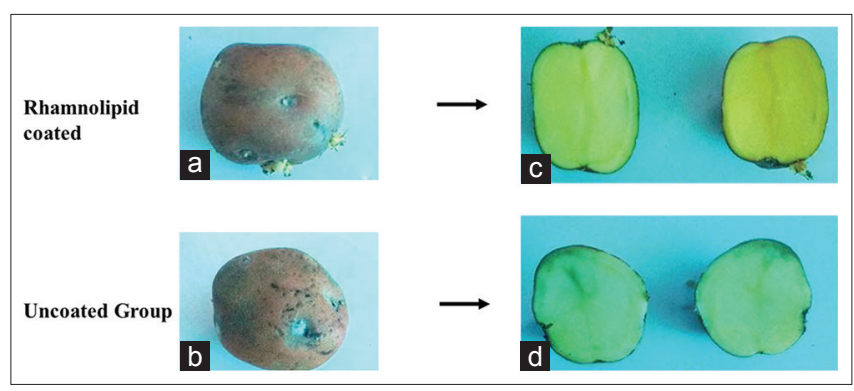

Fig. 6: In vivo antifungal activity of rhamnolipid by surface coating of potato against Fusarium solani (MTCC 350) after 15 days. Here, (a) is the rhamnolipid-coated potato after 15 days; (b) is showing the interior of the rhamnolipid-coated potato after 15 days; (c) is the uncoated potato after 15 days; (d) is showing the interior of the uncoated potato after 15 days

methods are being followed by agriculture practitioners to reduce the pre-harvesting and post-harvesting losses; however, their side effects, energy requirements, non-eco-friendly nature, and overall feasibility left the researcher to look for alternative methods.

Biosurfactants are amphiphilic molecules with some very useful properties which possess the great potential to be used in industries. In recent past, biosurfactants have been widely reported to exhibit significant antimicrobial activity against a variety of bacteria and fungi $[13,14]$. These reports greatly emphasize on the possible application of these biosurfactants in the development of various antimicrobial preparations. In past, biosurfactants have been reported to inhibit the growth of a variety of fungal species in various in vitro and vegetable-based in vivo models, thus suggesting its possible use in controlling the fungal spoilage in fruits and vegetables $[6,7]$. Therefore, this study was targeted to screen the bacterial biosurfactants for their potential to control fungal spoilage of vegetables. During this study, bacterial rhamnolipid biosurfactant exhibited significant antifungal activity in in vitro methods and its thing coating protected the vegetables from fugal spoilage for 15 days, thus increasing the life span of the vegetables significantly.

Similar kind of observations has been reported by other researchers where $B$. subtilis SPB1 lipopeptides biosurfactant controlled the dry rot potato tubers caused by F. solani in potato [6]. In addition to antimicrobial properties, in recent past, biosurfactants have also been reported to exhibit antiadhesive properties against a large number of microorganisms, thus also controlling the microbial attachment and growth on any surface over which they have been applied. One of the study reported the antiadhesive property of biosurfactant produced by lactobacilli against Candida albicans biofilm while in other study biosurfactant Lunasan produced by Candida sphaerica UCP 0995 
exhibited significant antiadhesive property against Streptococcus agalactiae, Pseudomonas aeruginosa, and Streptococcus sanguis $[15,16]$.

\section{CONCLUSION}

During this study, Pseudomonas sp. rhamnolipid biosurfactant exhibited significant antifungal activity against phytopathogen A. oryzae (MTCC 1846), F. solani (MTCC 350), and Curvularia sp. rhamnolipid coating $(1 \mathrm{mg} / \mathrm{ml})$ of the suitable vegetable protected them from fungal spoilage for up to 15 days at room temperature and thus increased their life span. Based on the above finding, it could be concluded that the Pseudomonas sp. rhamnolipid biosurfactant possesses significant antifungal activity and can be used for the development of coating material for the protection of vegetables from microbial spoilage.

\section{REFERENCES}

1. Barth M, Hankinson TR, Zhuang H, Breidt F. Microbiological spoilage of fruits and vegetables. In: Sperber WH, Doyle M, editors. Compendium of the Microbiological Spoilage of Foods and Beverages. $1^{\text {st }}$ ed. New York: Springer; 2009. p. 135-83.

2. Sharma D, Saharan BS. Functional characterization of biomedical potential of biosurfactant produced by Lactobacillus helveticus. Biotechnol Rep 2016;11:27-35.

3. Fatima Z, Saleemi M, Zia M, Sultan T, Aslam M, Rehman R, et al. Antifungal activity of plant growth-promoting rhizobacteria isolates against Rhizoctonia solani in wheat. Afr J Biotechnol 2009;8:219-25.

4. Okigbo RN. Biological control of postharvest fungal rot of yam (Dioscorea spp.) with Bacillus subtilis. Mycopathologia 2005;159:307-14.

5. Banat IM, Franzetti A, Gandolfi I, Bestetti G, Martinotti MG, Fracchia L, et al. Microbial biosurfactants production, applications and future potential. Appl Microbiol Biotechnol 2010;87:427-44.

6. Mnif I, Hammami I, Triki MA, Azabou MC, Ellouze-Chaabouni S,
Ghribi D. Antifungal efficiency of a lipopeptide biosurfactant derived from Bacillus subtilis SPB1 versus the phytopathogenic fungus, Fusarium solani. Environ Sci Pollut Res Int 2015;22:18137-47.

7. Mnif I, Grau-Campistany A, Coronel-León J, Hammami I, Triki MA, Manresa A, et al. Purification and identification of Bacillus subtilis SPB1 lipopeptide biosurfactant exhibiting antifungal activity against Rhizoctonia bataticola and Rhizoctonia solani. Environ Sci Pollut Res Int 2016;23:6690-9.

8. Ongena M, Jacques P. Bacillus lipopeptides: Versatile weapons for plant disease biocontrol. Trends Microbiol 2008;16:115-25.

9. Abdel-Mawgoud AM, Lépine F, Déziel E. Rhamnolipids: Diversity of structures, microbial origins and roles. Appl Microbiol Biotechnol 2010;86:1323-36.

10. Abalos A, Pinazo A, Infante MR, Casals M, Garcia F, Manresa A. Physicochemical and antimicrobial properties of new rhamnolipids produced by Pseudomonas aeruginosa AT10 from soybean oil refinery wastes. Langmuir 2001;17:1367-71.

11. Goswami D, Handique PJ, Deka S. Rhamnolipid biosurfactant against Fusarium sacchari - The causal organism of pokkah boeng disease of sugarcane. J Basic Microbiol 2014;54:548-57.

12. Gilman JC. A Manual of Soil Fungi. $1^{\text {st }}$ ed. New Delhi: Oxford and IBH Publishing Co.; 1967.

13. Singh AK, Rautela R, Cameotra SS. Substrate dependent in vitro antifungal activity of Bacillus sp. strain AR2. Microb Cell Fact 2014;13:67.

14. Gomaa EZ. Antimicrobial activity of a biosurfactant produced by Bacillus licheniformis strain M104 grown on whey. Braz Arch Biol Technol 2013;56:259-68.

15. Luna JM, Rufino RD, Sarubbo LA, Rodrigues LR, Teixeira JA, de Campos-Takaki GM. Evaluation antimicrobial and antiadhesive properties of the biosurfactant Lunasan produced by Candida sphaerica UCP 0995. Curr Microbiol 2011;62:1527-34.

16. Gomaa EZ. Antimicrobial and anti-adhesive properties of biosurfactant produced by lactobacilli isolates, biofilm formation and aggregation ability. J Gen Appl Microbiol 2013;59:425-36. 